\title{
IDENTIFICAÇ̃̃O DAS CARACTERÍSTICAS DEFINIDORAS DO DIAGNÓSTICO DE ENFERMAGEM EXCESSO DE VOLUME DE LÍQUIDOS
}

\author{
Heloísa Cristina Quatrini Carvalho Passos Guimarães* \\ Alba Lúcia Botura Leite de Barros** \\ Maria Gaby Rivero de Gutierrez***
}

GUIMARÃES, H.C.Q.C.P., BARROS, A.L.B.L.de, GUTIERREZ, M.G.R.de. Identificação das características definidoras do diagnóstico de enfermagem excesso de volume de líquidos. Rev.latino-am.enfermagem, Ribeirão Preto, v. 8, n. 2, p. 68-73, abril 2000.

Este estudo foi realizado com o objetivo de identificar as características definidoras do diagnóstico de enfermagem Excesso de Volume de Líquidos, proposto pela NANDA. Os dados foram coletados num hospital especializado em cardiologia, com uma amostra de 29 pacientes, cuja seleção foi realizada por duas enfermeiras peritas no assunto. Concluiu-se que houve concordância das duas peritas na identificação de nove pacientes com o diagnóstico em estudo e 20 sem o diagnóstico. As características definidoras identificadas aparecem em percentagens diferentes nos pacientes estudados.

UNITERMOS: diagnóstico de enfermagem

\section{INTRODUÇÃO}

No Brasil, começou-se a falar em diagnóstico de enfermagem na década de 70, com os trabalhos de Horta, mas somente nos anos 90 este assunto ganhou força, retomando-se os estudos e ampliando-se a utilização do diagnóstico como fase do processo de enfermagem. A definição de diagnóstico de enfermagem proposta pela NORTH AMERICAN NURSING DIAGNOSIS ASSOCIATION (NANDA) é de que se trata do julgamento clínico das respostas do indivíduo, família e da comunidade aos processos vitais ou aos problemas de saúde atuais ou potenciais, os quais fornecem a base para seleção das intervenções de enfermagem, para atingir resultados, pelos quais a enfermeira é a responsável (FARIAS et al., 1990).

ANGERAMI (1991) avalia que, ao elaborar o diagnóstico de enfermagem, o enfermeiro dá significado à coleta de dados, analisando e interpretando os achados e traçando o percurso de ação. Ao dar significado aos dados, o enfermeiro revela o profissional que é, sua visão de mundo, sua base teórica, sua inserção profissional. A intervenção proposta ao fenômeno que se apresenta, a realidade e os encaminhamentos de intervenção e avaliação de resultados são os indicadores da competência profissional no processo de cuidar e curar.

Segundo COLER (1992), a cultura do Brasil é tão diferente da norte-americana, que algumas características definidoras poderão não corresponder nos dois países. Este fato, por si só justificaria a necessidade de estudos de validação. Ainda a mesma autora refere que validação é o grau no qual alguma coisa mede o que ela deve medir e, neste contexto, todos os componentes da estrutura dos diagnósticos de enfermagem proposta pela NANDA poderiam ser validados.

Outros autores como FEHERING (1987); LEVIN et al. (1989); GORDON (1989, 1990); McKEIGEREM et al. (1990); MITCHELL (1991); COLER (1992), também enfocam a necessidade da validação dos diagnósticos de enfermagem.

A utilização prática dos diagnósticos de enfermagem propostos pela NANDA no exercício diário da nossa profissão no Instituto Dante Pazzaneze de Cardiologia (IDPC), a reflexão suscitada pelo contato com bibliografia a respeito deste tema, além das indagações oriundas da nossa prática, estimulou-nos a procurar desenvolver trabalho nesta área. Assim, dentre os diagnósticos propostos, decidimos aprofundar o estudo

\footnotetext{
* Doutoranda em Enfermagem pela UNIFESP. Mestre em Enfermagem na Saúde do Adulto pela UNIFESP. Enfermeira Pesquisadora do Instituto Dante Pazzanese de Cardiologia do Estado de São Paulo

** Doutor em Ciências pela Escola Paulista de Medicina. Professor Adjunto da Disciplina FEEMEC do Departamento de Enfermagem da UNIFESP. Diretora de Enfermagem do Hospital São Paulo - UNIFESP

*** Doutor em Enfermagem pela Escola de Enfermagem da Universidade de São Paulo. Professor Adjunto da Disciplina FEEMEC do Departamento de Enfermagem da UNIFESP
} 
sobre o "Excesso de Volume de Líquido", visando a sua validação, por ser este um dos mais freqüentes em pacientes cardiopatas.

O diagnóstico de enfermagem "Excesso de Volume de Líquido" está classificado no Padrão de Respostas Humanas Trocar que, segundo FARIAS et al. (1990), é definido como sendo "o estado em que o indivíduo experimenta aumentada retenção de líquidos e edema".

As características definidoras são o conjunto de sinais e sintomas que asseguram a presença de um determinado diagnóstico. São consideradas críticas aquelas que, sozinhas, são suficientes para confirmar o diagnóstico (MARIA, 1990).

O diagnóstico "Excesso de Volume de Líquido" tem como características definidoras: edema, infiltração, anasarca, ganho de peso, dispnéia, ortopnéia, ingestão maior que a excreta, $3^{a}$ bulha cardíaca, congestão pulmonar ao Raio $\mathrm{X}$ de tórax, ruídos adventícios (creptos), mudança no padrão respiratório, mudança no estado mental, hemoglobina e hematócrito baixos, variação na pressão arterial (PA), variação na pressão venosa central (PVC), variação na pressão de artéria pulmonar (PAP), ingurgitamento jugular, reflexo hepatojugular positivo, oligúria, variação na densidade específica da urina, azotemia, alteração de eletrólitos, inquietação e ansiedade.

Os fatores relacionados deste diagnóstico são: comprometimento dos mecanismos reguladores, excessiva ingestão de líquidos e excessiva ingestão de sódio.

Existem poucos trabalhos sobre a validação do diagnóstico de enfermagem "Excesso de Volume de Líquidos". DOUGHERTY (1993), no estudo em que faz um levantamento dos diagnósticos de enfermagem publicados no período de 1950 a 1993, cita apenas Sergent, que fez um estudo de validação deste diagnóstico em 1990, utilizando a técnica Delphi. Neste estudo foi constatado que as características definidoras: edema, infiltração, anasarca, ruídos adventícios (estertores creptantes e sibilos) congestão pulmonar ao Raio X de tórax, dispnéia, ortopnéia, ganho de peso, ingestão maior que excreta, oligúria, variação da densidade da urina, alteração da P.V.C, da PAP, da PA, ingurgitamento de jugular, reflexo hepato-jugular positivo, $3^{\mathrm{a}}$ bulha cardíaca e alteração de eletrólitos, obtiveram um score de 4,1 a 5,0 e foram consideradas como características maiores. As características menores, que atingiram um score de 3,1 a 4,0, foram as seguintes: inquietação, ansiedade, hematócrito e hemoglobina baixos, mudança no estado mental. Nenhuma das características deixou de ser considerada relevante.

Face ao exposto, indagamos: quais seriam as características definidoras propostas pela NANDA, que enfermeiras do IDPC utilizam, em sua prática diária, para definir ou estabelecer o diagnóstico enfermagem Excesso de Volume de Líquidos?
Este trabalho é parte integrante de um estudo sobre validação do Diagnóstico de Enfermagem "Excesso de Volume de Líquidos". Essa primeira etapa consiste da seleção, por duas enfermeiras especialistas, dos pacientes portadores ou não do referido diagnóstico.

\section{OBJETIVO}

Identificar quais das características definidoras, propostas pela NANDA (1989) para o diagnóstico em estudo, foram identificadas em maior e menor percentual, por duas enfermeiras peritas, para selecionar dois grupos de pacientes por elas definidos como portadores ou não deste diagnóstico.

\section{METODOLOGIA}

Este é um estudo exploratório de natureza descritiva, realizado no IDPC de Cardiologia, instituição governamental da cidade de São Paulo, especializado no atendimento de pacientes com doenças cardiovasculares, que tem como filosofia de trabalho a integração das atividades de assistência, ensino e pesquisa. Os dados foram coletados nas unidades de Recuperação Pós Operatória II, Pronto Socorro, Unidade Coronariana II, Unidade de Emergência e Enfermaria.

A amostra foi constituida de 29 pacientes que concordaram em participar do estudo e que, após análise das peritas foram divididos em dois grupos: com e sem o diagnóstico de enfermagem "Excesso de Volume de Líquidos". No primeiro grupo foram identificados 9 pacientes e no segundo 20, aos quais foi-lhes assegurado o anonimato e a liberdade de interromper o estudo se sentissem algum desconforto ou cansaço. Esses pacientes eram portadores de diferentes afecções cardíacas, tanto clínicas quanto cirúrgicas, internados nas unidades anteriormente citadas. A duas enfermeiras peritas tinham especialização em Enfermagem Médico-Cirúrgico, experiência na área de especialidade e na utilização dos Diagnósticos de Enfermagem conforme propostos pela NANDA, eram mestrandas em Enfermagem na Saúde do Adulto, e haviam cursado as disciplinas de Fisiologia Geral e Propedêutica Médica do programa curricular do curso de Medicina da Universidade Federal de São Paulo - Escola Paulista de Medicina, com o objetivo de complementar e aprofundar o conhecimento nestas áreas. $\mathrm{O}$ critério para determinar quais pacientes seriam incluídos nos grupos com e sem o diagnóstico foi o número de características definidoras coincidentes do diagnóstico em estudo, identificado nos pacientes que constituíram a amostra. Neste estudo, a quantidade de características consideradas para definir o diagnóstico foi seis ou mais, das coincidentes entre ambas peritas. A escolha deste número foi feita com base na experiência prática das peritas na identificação de características 
definidoras para determinar a presença do diagnóstico em estudo. Assim sendo, os pacientes que tiveram identificadas cinco ou menos características definidoras coincidentes entre ambas, bem como aqueles que, tendo seis ou mais características definidoras identificadas, porém não coincidentes entre as peritas, foram incluídos no grupo considerado sem o diagnóstico. Não foi estabelecido previamente quais características definidoras seriam incluídas dentre as seis coincidentes, para definir o diagnóstico devido a falta de especificação de características críticas, principais ou secundárias para este diagnóstico, na classificação da NANDA e para não induzir as peritas a identificarem, no paciente, aquelas características previamente selecionadas.

Os dados foram coletados nos períodos de outubro a novembro de 1994 e de março a abril 1995, pelas duas enfermeiras peritas que selecionaram os pacientes, com base na identificação das características definidoras, através do exame físico e análise do prontuário.

As peritas examinavam o mesmo paciente, separada e seqüencialmente, e assinalavam num formulário (Anexo 1) a presença ou ausência das características definidoras do diagnóstico em estudo. A seguir, os formulários eram confrontados e, com base nos critérios pré-estabelecidos para a formação dos grupos, os pacientes eram incluídos no grupo com e sem o diagnóstico. O tempo de duração do exame de cada paciente, foi de aproximadamente uma hora, sendo que os mesmos não manifestaram cansaço ou desconforto por serem submetidos a esta investigação.

Para a análise dos dados foram aplicados os seguintes testes estatísticos:

- Teste de Wilcoxon, (SIEGEL, 1975), foi utilizado para analisar a concordância entre as peritas quanto ao percentual de características definidoras identificadas do diagnóstico em estudo. Este teste foi aplicado separadamente para os grupos com e sem diagnóstico.

- Teste de Mann-Withney, (SIEGEL, 1975), foi utilizado para analisar o percentual e características definidoras identificadas nos grupos com e sem diagnóstico. Esta análise foi aplicada separadamente para as respostas de cada uma das peritas.

Em todos os testes, fixou-se em 0,05 ou $5 \%(\alpha<$ $0,05)$ o nível de rejeição da hipótese de nulidade, assinalando-se com asterisco os valores significantes.

\section{RESULTADOS E DISCUSSÃO}

Os resultados percentuais das características definidoras estudadas, bem como as suas médias, serão apresentados na Tabela 1. A discussão será feita simultaneamente à apresentação dos resultados.

Tabela 1 - Percentual das características definidoras do diagnóstico de enfermagem "Excesso de Volume de Líquidos", identificadas pela Perita 1 e Perita 2, nos grupos de pacientes considerados portadores ou não do diagnóstico. São Paulo, 1995

\begin{tabular}{|c|c|c|c|c|}
\hline Peritas & $\mathrm{COM}$ & DIAGNÓSTICo & SEM & DIAGNÓSTIC O \\
\hline características & $\begin{array}{c}\text { PERITAl } \\
\%\end{array}$ & $\begin{array}{c}\text { PERITA } 2 \\
\%\end{array}$ & $\begin{array}{c}\text { PERITAl } \\
\%\end{array}$ & $\begin{array}{c}\text { PERITA } 2 \\
\%\end{array}$ \\
\hline $\begin{array}{l}\text { edema } \\
\text { infiltração } \\
\text { anasarca } \\
\text { ganhode peso } \\
\text { dispnéia } \\
\text { ortopnéia } \\
\text { ingestäo = que excreta } \\
3^{\circ} \text { bulha } \\
\text { congestãopulmonar } \\
\text { ruídos adventícios } \\
\text { mudança padrão resp. } \\
\text { mudança estado mental } \\
\text { Hte Hb diminuidos } \\
\text { variação PA } \\
\text { variação da PVC } \\
\text { variação da PAP } \\
\text { ingurgitamentojugular } \\
\text { reflexp hepato jugular+ } \\
\text { oligúria } \\
\text { alteração densidade urina } \\
\text { azotemia } \\
\text { alteração conc. Eletrólitos } \\
\text { inquietação } \\
\text { ansiedade }\end{array}$ & $\begin{array}{c}55,5 \\
44,4 \\
22,2 \\
0 \\
44,4 \\
66,6 \\
77,7 \\
0 \\
0 \\
88,8 \\
77,7 \\
22,2 \\
55,5 \\
100 \\
55,5 \\
11,1 \\
33,3 \\
0 \\
44,4 \\
0 \\
66,6 \\
88,8 \\
22,2 \\
22,2\end{array}$ & $\begin{array}{c}55,5 \\
44,4 \\
11,1 \\
0 \\
66,6 \\
66,6 \\
55,5 \\
0 \\
33,3 \\
88,8 \\
88,8 \\
22,2 \\
33,3 \\
88,8 \\
66,6 \\
22,2 \\
44,4 \\
0 \\
11,1 \\
0 \\
33,3 \\
66,6 \\
22,2 \\
33,3\end{array}$ & $\begin{array}{c}30 \\
35 \\
10 \\
5 \\
15 \\
40 \\
50 \\
0 \\
0 \\
60 \\
5 \\
0 \\
50 \\
65 \\
40 \\
5 \\
15 \\
0 \\
25 \\
0 \\
40 \\
50 \\
5 \\
0\end{array}$ & $\begin{array}{l}55 \\
20 \\
0 \\
5 \\
25 \\
20 \\
40 \\
5 \\
15 \\
30 \\
55 \\
30 \\
35 \\
75 \\
50 \\
10 \\
10 \\
0 \\
5 \\
5 \\
5 \\
65 \\
10 \\
0\end{array}$ \\
\hline Média & 41,6 & 39,7 & 22,7 & 23,8 \\
\hline
\end{tabular}


TESTE DE WLCOXON

PERITA $1 \mathrm{X}$ PERITA 2

$\begin{array}{ll}\text { COM DIAG. } & \text { SEM DIAG. } \\ \text { Tcalc }=38,50 & \text { Tcalc }=113,50 \\ \text { Tcrit }=21,00 & \text { Tcrit }=59,00\end{array}$

TESTE DE MANN-WHITNEY

$Z_{\text {crit }}=1,96$

COM X SEM DIAG.

PERITA 1

$\mathrm{Zcalc}=1,99 *$

PERITA 2

$Z_{\mathrm{calc}}=1,98^{*}$

Aplicando o teste de Wilcoxon, nos dados apresentados na Tabela 1 , verificamos que não existe diferença significante entre os achados da perita 1 e da perita 2, em relação aos pacientes portadores ou não do diagnóstico em estudo. Além disso, o teste de MannWhitney, demonstra que a média percentual de características definidoras identificadas pelas peritas nos pacientes com o diagnóstico é significantemente maior que a média identificada nos pacientes sem o diagnóstico.

Constatamos também, na mesma tabela, que as características: edema, ortopnéia, ingestão maior que excreta, ruídos adventícios, mudança no padrão respiratório, variação na $\mathrm{PA}$, variação na $\mathrm{PVC}$ e alteração eletrolítica, foram as identificadas em percentuais que variam de 50 a $100 \%$, nos pacientes definidos pelas duas peritas como portadores do diagnóstico em estudo.

SERGENT et al. (1990), em seu estudo de validação deste diagnóstico pela técnica Delphi, considerou as características definidoras variação da PA, ruídos adventícios e ingestão maior que a excreta como características principais, estes achados são coincidentes com os do nosso estudo, quanto às características variação da PA e ruídos adventícios, pois as mesmas foram encontradas em percentuais superiores a $80 \%$, nos pacientes definidos pelas duas peritas como portadores do diagnóstico.

Verificamos também, na mesma tabela, que as características: anasarca, mudança no estado mental, variação na PAP e inquietação, obtiveram os menores percentuais de identificação nos mesmos pacientes.

Convém considerar ainda, que as características: ganho de peso, $3^{\text {a }}$ bulha, reflexo hepato-jugular positivo e alteração na densidade específica da urina não foram identificadas, por nenhuma das peritas. Este dado pode estar relacionado ao fato de que estes controles não são realizados rotineiramente nestas unidades, e também, com o preparo das peritas para identificar, especificamente, a $3^{\text {a }}$ bulha e o reflexo hepato jugular positivo.

No que diz respeito aos pacientes definidos pelas 2 peritas como não portadores do diagnóstico, podemos verificar na Tabela 1, que a característica: variação na PA foi identificada em percentuais situados entre 65 a $75 \%$ enquanto que as características variação na PAP, ingurgitamento da jugular, inquietação e ganho de peso foram encontradas em percentuais de $15 \%$ para baixo.

\section{CONCLUSÕES}

As conclusões que ora apresentamos, representam o resultado preliminar de um estudo mais amplo, sobre validação do diagnóstico de enfermagem “ Excesso de Volume de Líquidos".

No que diz respeito às características definidoras identificadas no presente estudo, concluimos que:

- Existe concordância entre as duas peritas na identificação dos pacientes com e sem diagnóstico.

- Os pacientes considerados pelas duas peritas como portadores do diagnóstico, apresentam maior percentagem de características definidoras, comparativamente aos identificados pelas mesmas como não portadores.

Nos pacientes considerados pelas peritas como portadores do diagnóstico em estudo:

- As características definidoras identificadas como maiores, ou seja, em percentuais que variam de 80 a $100 \%$ foram ruídos adventícios e variação da PA;

- As características definidoras: edema, ortopnéia, ingestão maior que excreta; mudança no padrão respiratório, variação na PVC e alteração nos eletrólitos, foram identificadas em percentuais que variam de 50 a $79 \%$, podendo ser consideradas menores.

Nos pacientes indicados pelas duas peritas como não portadores do diagnóstico:

- A característica definidora variação na PA foi identificada em percentuais que variam de 65 a $75 \%$ e, - As características definidoras, ganho de peso, variação na PAP, ingurgitamento da jugular e inquietação obtiveram percentuais de identificação situados entre 5 a $10 \%$.

\section{IDENTIFICATION OF DEFINING CHARACTERISTICS OF THE NURSING DIAGNOSIS FLUID VOLUME EXCESS}

This study aimed at verifying the defining characteristics of the nursing diagnosis "Fluid Volume Excess" proposed by NANDA. Data collection was carried out within a specialized Cardiology health care setting where the 29 patients were selected by two expert nurses. The conclusion showed consonance of opinions between the two professionals in regard to the identification 
of nine patients with diagnosis and 20 without this type of diagnosis. The identified defining characteristics appeared in different percentages considering the studied patients.

\section{IDENTIFICACIÓN DE LAS CARACTERISTICAS DEFINIDORAS DEL DIAGNÓSTICO DE ENFERMERÍA EXCESSO DE VOLUMEN DE LIQUIDOS}

Este estudio fue llevado a cabo con el objetivo de validar el diagnóstico de enfermería "Exceso de Volumen de Líquidos “. Para esto se buscó verificar la existencia o no de concordancia entre enfermeras de un hospital especializado en cardiología en lo que respecta a la identificación de las características definitorias utilizadas para determinar dicho diagnóstico, tal como es propuesto por la Asociación Norteamericana de Diagnósticos de Enfermería. El estudio fue realizado con 29 pacientes evaluados, en un primer momento, por dos enfermeras especialistas. Los resultados mostraron que las dos enfermeras concordaron en la indicación de presencia del diagnóstico objeto de estudio, en 9 pacientes y de ausencia en 20. Las características definitorias que determinaban la presencia o ausencia del diagnóstico se presentaron en diferentes porcentajes en los pacientes estudiados.

\section{ANEXO 1}

NOME DO PACIENTE:

LEITO: _ RG: _ _ DATA DE INTERNAÇÃO: $/$
IDADE:
DATA DA ENTREVISTA: ____ HORA:
DIAGNÓSTICOS: EXCESSO DE VOLUME DE
LÍQUIDOS

Identifique com um $\mathbf{X}$ quais as características que estão presentes na prática diária no paciente examinado por você. Após a identificação destas, defina a presença ( ) ou ausência ( ) do diagnóstico.

\section{CARACTERÍSTICAS DEFINIDORAS}

\section{1 - EDEMA}

ausente ( )

presente ( )

\section{2 - INFILTRAÇÃO} ausente ( )

$$
\text { presente ( ) }
$$

\section{3 - ANASARCA}

ausente ( )

presente ( )

\section{4 - GANHO DE PESO}

ausente ( )

presente ( )

\section{5 - DISPINÉIA}

ausente ( )

\section{6 - ORTOPNÉIA}

ausente ( ) presente ( )

\section{7 - INGESTÃO MAIOR QUE EXCRETA}

ausente ( ) presente ( )

8 - $3^{\text {a }}$ BULHA

ausente ( ) presente ( )

\section{9 - CONGESTÃO PULM. (RX TÓRAX)}

ausente ( ) presente ( )

10 - RUÍDOS ADVENTÍCIOS; CREPTOS

ausente ( ) presente ( )

11 - MUD. NO PADRÃO RESPIRATÓRIO ausente ( ) presente ( )

12 - MUD. NO ESTADO MENTAL ausente ( ) presente ( )

\section{3 - Ht E Hb BAIXOS}

ausente ( ) presente ( )

14 - VARIAÇÃO NA P.A ausente ( ) presente ( )

15 - VARIAÇÃO NA P.V.C ausente ( ) presente ( )

16 - VARIAÇÃO NA P.A.P

ausente (

presente ( ) 
17 - INGURGITAMENTO DE JUGULAR

ausente ( ) presente ( )

18 - REFLEXO HEPATO-JUGULAR +

ausente ( ) presente ( )

19 - OLIGÚRIA

ausente ( ) presente ( )

20 - VAR. NA DENSIDADE NA URINA

ausente ( )

presente ( )

\section{REFERÊNCIAS BIBLIOGRÁFICAS}

01. ANGERAMI, E.L.S. O que é diagnóstico de enfermagem. In: SIMPÓSIO NACIONAL SOBRE DIAGNÓSTICO DE ENFERMAGEM,1, São Paulo, 1991. Anais. São Paulo, 1991. p. 233.

02. COLER, M.S. Pesquisa e validação dos diagnósticos de enfermagem. In: SIMPÓSIO NACIONAL SOBRE DIAGNÓSTICOS DE ENFERMAGEM, 2 e SEMINÁRIO PARAIBANO DE DIAGNÓSTICO DE ENFERMAGEM, 3, João Pessoa, 1992. Anais. João Pessoa, 1992. p. 189.

03. DOUGHERTY, C.M. et al. Conceptual and researchbased validation of nursing diagnoses: 1950 a 1993. Nurs. Diag., v. 4, n. 1, p. 156-215, 1993.

04. FARIAS, J.N. et al. Diagnóstico de enfermagem: uma abordagem conceitual e prática. João Pessoa: Santa Marta, 1990.

05. FEHERING, R. Methods to validate nursing diagnosis Heart \& Lung, v. 16, n. 6, p. 625-9, 1987.

06. GORDON, M. Proceedings of the invitational conference on research methods for validating nursing diagnoses In: MONOGRAPH OF THE INVITATIONAL CONFERENCE ON RESEARCH METHODS OF VALIDATING NURSING DIAGNOSIS. Palm Springs, 1989.

\section{1 - AZOTEMIA}

ausente ( )

presente ( )

22 - ALTERAÇÃO DE ELETRÓLITOS

ausente ( )

presente ( )

23 - INQUIETAÇÃO

ausente ( ) presente ( )

24 - ANSIEDADE

ausente ( ) presente ( )
07. GORDON, M. Toward theory - based diagnostic categories. Nurs.Diag., v. 1, n. 1, p. 5-11, 1990.

08. LEVIN, R.F. et al. Diagnostic content validity of nursing. Image J. Nurs. Scholarship, v. 21, n. 1, p. 40-4, 1989.

09. MARIA, V.L.R. Preparo das enfermeiras para utilização dos diagnósticos de enfermagem: relato de experiência. São Paulo, 1990. 130p. Dissertação (Mestrado) - Escola de Enfermagem, Universidade de São Paulo.

10. McKEIGHEN, R.J; FANN, P.A.M.; DICKEL, C.A. Bathing / higiene self-care. Deficit: defining characteristics and related factors: across age groups in an acute care setting. Nurs.Diag., v. 1, n. 4, p. 155-161, 1990.

11. MITCHELL, G. Nursing diagnosis: an ethical analysis. Image J. Nurs. Scholarship, v. 23, p. 99-103, 1991.

12. SERGENT, E. et al. Diagnostic content validity of fluid volum excess: a construct replication. In: NORTH AMERICAN NURSING DIAGNOSES ASSOCIATION Nursing diagnoses proceedings of ninth conference. Philadelphia: Lippincott, 1990.

13. SIEGEL, S. Estatística no paramétrica. México: Trillos, 1975. 